\title{
Hospital-acquired fever in oriental medical hospitals
}

\author{
Soo-youn Moon ${ }^{1}$, Ki-Ho Park', Mi Suk Lee ${ }^{1}$ and Jun Seong Son ${ }^{2^{*}}$ (D
}

\begin{abstract}
Background: Traditional Oriental medicine is used in many Asian countries and involves herbal medicines, acupuncture, moxibustion, and cupping. We investigated the incidence and causes of hospital-acquired fever (HAF) and the characteristics of febrile inpatients in Oriental medical hospitals (OMHs).

Methods: Patients hospitalized in two OMHs of a university medical institute in Seoul, Korea, were retrospectively reviewed from 2006 to 2013. Adult patients with HAF were enrolled.

Results: There were 560 cases of HAF (5.0\%). Infection, non-infection, and unknown cause were noted in 331 cases (59.1\%), 109 cases (19.5\%), and 120 cases (21.4\%) of HAF, respectively. Respiratory tract infection was the most common cause (51.2\%) of infectious fever, followed by urinary tract infection. Drug fever due to herbal medicine was the most common cause of non-infectious fever (53.1\%), followed by procedure-related fever caused by oriental medical procedures. The infection group had higher white blood cell count (WBC) $\left(10,400 / \mathrm{mm}^{3} \mathrm{vs} .7000 / \mathrm{mm}^{3}, p<0.001\right)$ and more frequent history of antibiotic therapy ( $29.6 \%$ vs. $15.1 \%, p<0.001)$. Multivariate analysis showed that older age (odds ratio (OR) 1.67, 95\% confidence interval (C.I.) 1.08-2.56, $p=0.020$ ), history of antibiotic therapy (OR 3.17, C.I. $1.85-5.41, p<0.001$ ), and WBC > 10,000/ $\mathrm{mm}^{3}$ (OR 2.22, C.I. 1.85-3.32, $p<0.001$ ) were associated with infection.
\end{abstract}

Conclusions: Compared to previous studies on HAF in Western medicine, the incidence of HAF in OMHs was not high. However, Oriental medical treatment does play some role in HAF. Fever in patients with history of antibiotic therapy, or high WBC was more likely of infectious origin.

Keywords: Fever, Hospital-acquired, Traditional oriental medicine, Etiology

\section{Background}

Fever is a common clinical event in hospitalized patients. Although fever is frequently suspected and proven to be related to infections, diverse etiologies may account for fever in hospitalized patients.

Hospital-acquired febrile illness is defined as a fever occurring at least $48 \mathrm{~h}$ after hospital admission [1]. The prevalence of hospital-acquired febrile illness has been estimated at $2 \%$ to $31 \%$ for medical inpatients [2]. There have been studies on the etiologies of fever in elderly patients, solid organ transplant recipients, cancer patients, and neutropenic hosts [3-7]. Fever was attributed to infection in 37\% to $74 \%$ of patients, whereas a non-infectious etiology was identified in $3 \%$ to $52 \%$ of patients [1]. The most common

\footnotetext{
* Correspondence: isonjs@naver.com

${ }^{2}$ Division of Infectious Diseases, Department of Internal Medicine, Kyung Hee University Hospital at Gangdong, 892, Dongnam-ro, Gangdong-gu, Seoul, South Korea

Full list of author information is available at the end of the article
}

infectious causes included urinary tract infection, pneumonia, sinusitis, and bloodstream infection [1]. The most common non-infectious causes were procedure-related, malignancies, and ischemic conditions [8].

Oriental traditional medicine has been practiced for a very long time in Korea, China, Japan and throughout Southeast Asia, including Vietnam, Thailand, and Tibet. It is also currently practiced as a part of alternative medicine in Western countries. Traditional Oriental medicine is part of medical practice in Korea, and there are many hospitals in which traditional Oriental medical doctors practice traditional medicine for hospitalized patients. In Korea, patients with cerebrovascular accident and elderly patients tend to seek oriental medical care more often than other patients [9].

Our medical centers are composed of a medical hospital, oriental medical hospital (OMH), and dental hospital. Many patients are hospitalized for traditional medical treatment, and some patients develop fever. When patients in the 
OMH develop fever, many are referred to the medical hospital for evaluation and treatment of fever. There has been no data on the incidence or etiology of fever in these patients. As far as we know, this is the first study on hospitalacquired febrile illness in OMHs.

This study was designed to identify the characteristics of febrile inpatients, causes of fever, and clinical outcomes of fever in OMHs and to identify the risk factors of febrile illness of infectious cause in these patients.

\section{Methods}

This retrospective study was performed at two OMHs of a university medical institute in Seoul, Korea. The medical institute consists of two medical hospitals, two OMHs, and two dental hospitals. The study protocol was approved by institutional review board (IRB) of Kyung Hee University Hospital at Gangdong (IRB No. 2016-03-008). Informed consent from the patients was waived by IRB.

Patients hospitalized in the OMHs from June 2006 to June 2013 were retrospectively reviewed via electronic medical records by two infectious diseases specialists. Patients age 18 years or older were screened. Adult patients with axillary body temperature $\geq 37.8^{\circ} \mathrm{C}$ after $48 \mathrm{~h}$ of hospitalization were enrolled. If a patient was transferred from another $\mathrm{OMH}$ where he or she was admitted for more than $48 \mathrm{~h}$ and where fever started within $48 \mathrm{~h}$ of hospitalization, these fevers were also considered hospital-acquired. If a patient was transferred from a medical hospital or long-term care facility and fever had started within $48 \mathrm{~h}$ of hospitalization to the $\mathrm{OMH}$, he or she was excluded.

Demographic characteristics, clinical features, laboratory data, and treatment history were collected by review of medical records. Infection was defined using criteria proposed by the Centers for Disease Control and Prevention [10].

Fever was considered procedure-related if there was a transient temperature elevation during the $48 \mathrm{~h}$ period following an invasive procedure and no evidence of infection elsewhere [1]. Drug fever was defined by diagnostic criteria adapted from those described by Young et al. [11] Patients with fever accompanied by drug rash were included if the fever resolved after discontinuing the offending drug. If patients with acute intra-cranial hemorrhage (ICH) or acute cerebral infarction developed fever with no other certain fever focus, the fever was considered related to the ICH or cerebral infarction. If patients had massive gastro-intestinal bleeding and no other obvious fever focus, the fever was considered related to gastrointestinal bleeding. If a fever was observed after transfusion of blood products such as packed RBC or platelet concentrates and no other cause was found, the fever was considered related to the transfusion. If a fever was observed in a severely dehydrated patient and it disappeared after adequate hydration, the fever was considered to be caused by dehydration. When there was unexplained fever in advanced cancer patients or patients with hematologic malignancy, the fever was considered a cancer fever.

If the etiology was uncertain or data was insufficient to determine a cause, the fever was defined as unknown. Defervescence was defined as peak body temperature below $37.3{ }^{\circ} \mathrm{C}$ for more than 2 days.

McCabe classification was used to evaluate the severity of underlying illnesses [12].

SPSS for Windows version 11.0 (SPSS Inc., Chicago, IL, USA) was used for statistical analysis. Student's t-test and Chi-square test were used for univariate analysis, and a logistic regression test was used for multivariate analysis. Factors with statistical significance in univariate analysis were chosen for multivariate analysis. $P$ values $<0.05$ were considered significant.

\section{Results}

A total of 11,207 adult patients were hospitalized in the OMHs during the study period. Among those 11,207 patients, 560 cases with hospital-acquired febrile events were identified (5.0\%).

Infection was identified as the cause of fever in 331 cases (59.1\%). In 179 cases (32.0\%), non-infectious causes of fever were identified (Table 1). There were 50 cases $(8.9 \%)$ of fever with unknown etiology. Among patients with fever caused by infections, respiratory tract infection was the most common cause (166 cases, $51.2 \%$ ), followed by urinary tract infection (99 cases, 30.6\%) and intra-abdominal infection (39 cases, 12.1\%) (Table 1). Among patients with respiratory infection, aspiration pneumonia was the most common cause. Among patients with non-infectious fever, drug fever was the most common cause (101 cases, 56.4\%), followed by procedure-related fever (24 cases, $13.4 \%)$ and cancer fever (23 cases, 12.8\%). Among 101 patients with drug fever, herbal medicine was the most common cause (95 cases, 94.1\%), while antibiotics or bisphosphonate were the cause of drug fever in 6 patients.

Among 11,207 adult patients hospitalized in OMHs, 10,880 were treated with herbal medicine, and 8125 underwent oriental procedures. There were 8040 patients who received acupuncture, 6585 patients who received moxibustion, and 4267 patients who received cupping. Ninety-five patients developed fever due to herbal medicine, 8 patients developed fever due to acupuncture, 7 developed fever patients due to moxibustion, and 1 patient developed fever due to cupping (Table 2). Overall incidence of procedure-related fever caused by invasive oriental procedures is $2.9 \%$ (16 episodes), while incidence of procedure-related fever caused by western medical procedures is $1.4 \%$ (8 episodes).

Table 3 shows the patient characteristics of febrile patients in the OMHs. The mean age of these patients was $61.4 \pm$ 15.2 years, and $49.6 \%$ of the patients were male. Patients with fever of infectious origin (infection group) were older than patients with fever of non-infectious origin (non-infection 
Table 1 Etiology of Hospital-acquired Fever in OMHs

\begin{tabular}{ll}
\hline Category & No. (\%) \\
\hline Infection & $331(59.1)$ \\
Respiratory tract & $166(51.2)$ \\
Urinary tract & $99(30.6)$ \\
Intra-abdominal & $39(12.1)$ \\
Skin and soft tissue & $18(3.2)$ \\
Primary bacteremia/fungemia & $10(3.1)$ \\
Central nervous system & $2(0.6)$ \\
Bone \& joint & $2(0.6)$ \\
Other & $2(0.6)$ \\
Non-infection & $179(32.0)$ \\
Drug fever & $101(56.4)$ \\
Post-procedure & $24(13.4)$ \\
Cancer fever & $23(12.8)$ \\
Transfusion related & $7(3.9)$ \\
Dehydration related & $3(1.7)$ \\
ICH related central fever & \\
Other & $2(1.1)$ \\
Unknown & $19(10.6)$ \\
\hline
\end{tabular}

${ }^{\mathrm{a}} \mathrm{CH}$ intracranial hemorrhage

group) $(63.7 \pm 15.3$ years vs. $58.0 \pm 14.7$ years, $p<0.001)$. More patients in the infection group had history of CVA (54.1\% vs. $35.2 \%, p<0.001$ ). There were more patients with malignancies in the non-infection group (55.9\% vs. $32.9 \%, p$ $<0.001)$. There was no statistical difference in the frequency of previous admission history between the two groups. Among 560 patients with fever, 506 (90.4\%) were treated with herbal medicine. Invasive oriental procedures, such as acupuncture, moxibustion, and cupping, were performed in 532 patients (95.0\%). Patients in the infection group had more frequent history of antibiotic treatment before fever onset (29.6\% vs. $15.1 \%)$. There was no significant difference in peak body temperature or fever duration between the infection group and non-infection group. WBC count was higher in the infection group (median 10,400/mm3 vs. median 7000/mm3, $p<0.001$ ); however, CRP level was not different between the two groups (Table 3).

More patients with infectious fever received consultations for evaluation and management of fever (72.8\% vs. 26.3\%, $p<0.001)$. Antibiotics were prescribed in 302 cases $(53.9 \%)$. More patients in the infection group were treated with antibiotics (73.7\% vs. $25.1 \%, p<0.001$ ), and the duration of antibiotic therapy was also longer in the infection group (median 9 days vs. median 5.5 days, $p=0.002$ ). Overall 30day mortality rate was $9.6 \%$ (Table 4 ).

Table 5 shows multivariate analysis for risk factors associated with infectious origin in febrile illnesses. Patients age over 65 years developed fever of infectious origin 1.666 times more often (95\% confidence interval (C.I.); 1.082-2.564, $p=$ 0.020 ) than younger patients. When patients had a history of antibiotic use, fever of infectious origin was 3.166 times more likely (95\% C.I.; $1.852-5.413, p<0.001)$. Fever in the patients with WBC higher than $10,000 / \mathrm{mm}^{3}$ were 2.223 times more likely to be caused by infection (95\% C.I.; 1.486-3.324, $p<$ 0.001 ). In patients with cancer or history of chemotherapy, the chance of infectious fever decreased 0.263 times (95\% C.I.; 0.116-0.594, $p=0.001$ ) and 0.507 times (95\% C.I.; $0.289-0.0887, p=0.017)$, respectively.

\section{Discussion}

Oriental traditional medicine in East Asia, including Korea, China, and Japan, uses acupuncture, moxibustion, cupping, herbal medicines, and manual therapies [13]. In Korea, traditional Oriental medicine is called Hanbang and is an inseparable component of Korean culture and Korean medical services [9]. Traditional Oriental medicine is included in the national health care system in Korea. National policies have been developed based on its historical and cultural background in a way that differs from its use as a complementary and alternative medicine in Western society [14]. Korea has the highest percentage (15.3\%) of traditional Oriental medical doctors in hospitals and clinics in East Asia, followed by mainland China (12.6\%) and the Taiwan region (9.7\%) [14] Korean patients with cerebrovascular accidents ( $\mathrm{ICH}$ or cerebral infarction), advanced cancer, facial palsy, and old age tend to seek traditional Oriental medicine more often than patients with other acute illnesses [9]. Because traditional Oriental medicine uses different treatment modalities than Western medicine, the etiology of fever might be different. As far as we know, this is the first study on the incidence and etiologies of hospital-acquired fever in patients hospitalized at OMHs.

Table 2 Incidence of drug fever and procedure-related fever among patients receiving herbal medicine and/or oriental procedures among patients admitted to OMHs $(n=11,207)$

\begin{tabular}{llll}
\hline Risk factor & No. of patients with risk factors & No. of patients with risk factors who developed fever & Percentage \\
\hline Herbal medicine & 10,880 & 95 & 0.87 \\
Invasive oriental medical procedures & 8125 & 16 & 0.20 \\
Acupuncture & 8040 & 8 & 0.10 \\
Moxibustion & 6585 & 7 & 0.11 \\
Cupping & 4267 & 1 & 0.02 \\
\hline
\end{tabular}


Table 3 Characteristics of Patients with HAF in OMH by Etiology

\begin{tabular}{|c|c|c|c|c|}
\hline & $\begin{array}{l}\text { Total } \\
(n=560)\end{array}$ & $\begin{array}{l}\text { Infection } \\
(n=331)\end{array}$ & $\begin{array}{l}\text { Non-infection } \\
(n=179)\end{array}$ & $p$ \\
\hline \multicolumn{5}{|l|}{ Demographics } \\
\hline Age (years) & $61.4 \pm 15.2$ & $63.7 \pm 15.3$ & $58.0 \pm 14.7$ & $<0.001$ \\
\hline Male sex & $278(49.6)$ & $169(51.1)$ & $81(45.3)$ & 0.228 \\
\hline \multicolumn{5}{|l|}{ Underlying diseases } \\
\hline Diabets mellitus & $141(25.2)$ & $90(27.2)$ & $40(22.3)$ & 0.243 \\
\hline Hypertension & $234(41.8)$ & $161(48.6)$ & $60(33.5)$ & 0.001 \\
\hline Cerebrovascular accident & $251(44.8)$ & $179(54.1)$ & $63(35.2)$ & $<0.001$ \\
\hline Solid cancer & $246(43.9)$ & $109(32.9)$ & $100(55.9)$ & $<0.001$ \\
\hline Metastatic cancer & $205(36.6)$ & $88(26.6)$ & $85(47.5)$ & $<0.001$ \\
\hline \multicolumn{5}{|l|}{ Healthcare- associatied conditions } \\
\hline Receipt of anticancer chemotherapy & $113(20.2)$ & $40(12.1)$ & $56(31.3)$ & $<0.001$ \\
\hline Central venous catheter & $68(12.1)$ & $43(13.0)$ & $21(11.7)$ & 0.682 \\
\hline Urinary catheter & $111(19.8)$ & 79 (23.9) & $27(15.1)$ & 0.022 \\
\hline Percutaneous drainage & $68(12.1)$ & $47(14.2)$ & $20(11.2)$ & 0.410 \\
\hline Previous operation & 76 (13.6) & $47(14.2)$ & $21(11.7)$ & 0.434 \\
\hline Previous intervention & $24(4.3)$ & $14(4.2)$ & $9(5.0)$ & 0.678 \\
\hline Previous admission & $414(74.0)$ & $250(75.5)$ & $134(74.9)$ & 0.867 \\
\hline Previous antibiotics & $128(22.9)$ & $98(29.6)$ & $27(15.1)$ & $<0.001$ \\
\hline \multicolumn{5}{|l|}{ Concurrent treatment } \\
\hline Herbal medicine & $506(90.4)$ & $310(93.7)$ & $161(89.9)$ & 0.132 \\
\hline Invasive Oriental medical procedure & $532(95.0)$ & $314(94.9)$ & $171(95.5)$ & 0.739 \\
\hline Acupuncture & $502(89.6)$ & $302(91.2)$ & $161(89.9)$ & 0.630 \\
\hline Cupping & $97(17.3)$ & $56(17.0)$ & 35 (19.6) & 0.468 \\
\hline Moxibustion & $417(74.5)$ & $242(73.3)$ & $138(77.1)$ & 0.352 \\
\hline $\mathrm{SIRS}^{\mathrm{a}}$ (severe or shock) & $47(8.4)$ & $28(8.5)$ & $14(7.8)$ & 0.788 \\
\hline \multicolumn{5}{|l|}{ Laboratory findings at the onset of fever } \\
\hline White Blood Cell(/mm³) & $9550(100-32,500)$ & $10,400(800-32,500)$ & $7000(100-24,000)$ & $<0.001$ \\
\hline Neutropenia $\left(\mathrm{WBC}<1000 / \mathrm{mm}^{3}\right)$ & $5(0.9)$ & $3(1.0)$ & $2(1.3)$ & 0.662 \\
\hline C-reactive protein & $5.3(0.0-39.7)$ & $5.4(0.0-39.7)$ & $4.2(0.0-22.5)$ & 0.767 \\
\hline \multicolumn{5}{|l|}{ Fever pattern } \\
\hline Peak body temperature $\left({ }^{\circ} \mathrm{C}\right)$ & $38.5 \pm 0.6$ & $38.6 \pm 0.6$ & $38.4 \pm 0.6$ & 0.151 \\
\hline Duration of fever & $1.0(0.0-18.0)$ & $1.0(0.0-18.0)$ & $1.0(0.0-17.0)$ & 0.263 \\
\hline No defervescence & $43(7.7)$ & $23(6.9)$ & $12(6.7)$ & 0.917 \\
\hline
\end{tabular}

${ }^{a}$ SIRS systemic inflammatory response syndrome

Table 4 Management and clinical outcome of febrile patients in $\mathrm{OMH}$

\begin{tabular}{lllll}
\hline & Total $(\mathrm{n}=560)$ & Infection $(\mathrm{n}=331)$ & Non-infection $(\mathrm{n}=179)$ & $\mathrm{p}$ \\
\hline Medical consult & $297(53.0)$ & $241(72.8)$ & $47(26.3)$ & $45(25.1)$ \\
Antibiotics & $302(53.9)$ & $244(73.7)$ & $5.5(1-21)$ & $<0.001$ \\
Duration of antibiotics & $8.0(0.0-40.0)$ & $9(1-40)$ & $31(17.3)$ & 0.001 \\
Antipyretics & $112(20.0)$ & $72(21.8)$ & $4(2.2)$ & 0.339 \\
Surgical treatment & $7(1.3)$ & $3(0.9)$ & $14(8.9)$ & 0.248 \\
30-day mortality & $46 / 477(9.6)$ & $21(7.6)$ & 0.616 \\
\hline
\end{tabular}


Table 5 Factors associated with infectious origin in febrile illness in $\mathrm{OMHs}$

\begin{tabular}{|c|c|c|c|c|}
\hline & Univariate analysis & $p$ & Multivariate analysis & $p$ \\
\hline & OR (95\% C.I.) & & OR (95\% C.I.) & \\
\hline Age $>65$ & $2.250(1.544-3.277)$ & $<0.001$ & $1.666(1.082-2.564)$ & 0.020 \\
\hline Hypertension & $1.878(1.287-2.741)$ & 0.001 & & \\
\hline Cerebrovascular accident & $2.168(1.490-3.156)$ & $<0.001$ & & \\
\hline Urinary catheter & $1.765(1.091-2.855)$ & 0.021 & & \\
\hline Previous antibiotic & $2.368(1.475-3.798)$ & $<0.001$ & $3.166(1.852-5.413)$ & $<0.001$ \\
\hline WBC $>10,000$ & $3.730(2.415-5.760)$ & $<0.001$ & $2.223(1.486-3.324)$ & $<0.001$ \\
\hline Solid cancer & $0.330(0.232-0.468)$ & $<0.001$ & $0.263(0.116-0.594)$ & 0.001 \\
\hline Metastatic cancer & $0.347(0.243-0.495)$ & $<0.001$ & & \\
\hline Recipient of anticancer chemotherapy & $0.294(0.191-0.452)$ & $<0.001$ & $0.507(0.289-0.0887)$ & 0.017 \\
\hline
\end{tabular}

In this study, incidence of HAF was $5.2 \%$, lower than that of other studies [2, 15]. Infection accounted for $59.1 \%$ of HAF among patients hospitalized in OMHs. In Arbo's study of HAF, infection accounted for $56.0 \%$ of fever, which is similar to our results [1]. Chang's study on liver transplant recipients showed a higher incidence of infection (78.0\%) [3]. In cancer patients, infection, non-infectious causes, and fever of unknown origin represented $67.0 \%, 23.0 \%$, and $10.0 \%$ of cases, respectively [7]. The respiratory tract was the most frequently involved site in cancer patients, [7] similar to our results.

In OMHs, more patients with solid cancer $(55.9 \%$ vs. $32.9 \%, p<0.001)$ and history of anti-cancer chemotherapy $(31.3 \%$ vs. $12.1 \%, p<0.001)$ have non-infectious fever. In these patients, moxibustion was more commonly used, and herbal medication was less commonly prescribed without statistical significance. Moxibustion was more commonly used in the non-infection group, while herbal medicine was more frequently prescribed to patients in the infection group. There were more patients with cancer in the non-infection group than in the infection group. Moxibustion was more frequently used in the non-infection group for pain relief in cancer patients. Some studies have shown that moxibustion is effective in reducing pain associated with osteoarthritis and herpes zoster [16]. Herbal medicine is a main treatment modality in traditional Oriental medicine and is prescribed to most patients. Unlike Western medicine, Oriental herbal medicine can only be administered by oral route. Some patients with advanced cancer were unable to orally take medication, so herbal medicine could not be prescribed.

Drug fever accounted for $56.4 \%$ of non-infectious fever in our study. In Cunha's studies on fever in a neurosurgical intensive care unit and intensive care unit, drug fever occurred in approximately $10.0 \%$ of patients $[17,18]$. In Toussaint's study on fever in cancer patients, drug fever accounted for $18.0 \%$ of fever not attributed to infection (non-infectious fever and fever of unknown origin together), which is lower than our study result [7]. In Oisumi's study on drug fever caused by antibiotics, drug fever was recognized in $13.1 \%$ of 390 patients receiving parenteral antibiotic therapy for pulmonary infections. Drugs have been estimated to cause $10.0-15.0 \%$ of adverse events and $3.0-5.0 \%$ of drug fever in hospitalized patients in the US [19].

Procedure-related fever in Western medicine was about $1.5 \%$ to $5.9 \%,[2,7]$ while incidence of procedurerelated fever in our $\mathrm{OMH}$ was $4.2 \%$, which was not different from other studies. Among the procedure-related fever in our $\mathrm{OMH}$, fever related to invasive oriental procedure was higher than that related to western medical procedures (2.9\% vs. $1.4 \%)$.

Old age, history of antibiotic use, and high WBC were associated with infection. Peak body temperature was not associated with infection in this study. In Trivalle's study on hospital-acquired febrile illness in the elderly, the number of invasive procedures preceding a febrile episode was a significant predictor of infection [2]. Other studies have shown that higher maximum temperature or higher peak WBC was associated with an infectious etiology for fever [8].

This study has some limitations. First, it was a retrospective study performed in OHMs. Culture and imaging studies are not performed in every patient with HAF. Therefore, some patients with infection may not have been identified and classified with unknown fever. Second, this study was performed at two OMHs of a university teaching hospital. In our hospitals, patients with advanced cancer or cerebrovascular attack are common. Patient characteristics and severity of underlying illness may be different from those of other OMHs.

\section{Conclusions}

In this study, incidence of HAF was not higher in OMHs, [2, 15] and infection was the most common cause of HAF. Fever in patients with history of antibiotic treatment and high was more likely of infectious origin. 
Herbal medicine and invasive oriental medical procedures do play some role in HAF as herbal medicine was the most common cause of drug fever and invasive oriental medical procedures caused procedure related fever more frequently than Western medical procedures in OMHs.

To identify the etiology of HAF in OMHs and the risk factors of fever of infectious origin, further multicenter study is suggested.

\section{Abbreviations}

C.I.: Confidence interval; CRP: C-reactive protein; HAF: Hospital-acquired fever; ICH: Intra-cranial hemorrhage; IRB: Institutional review board; OMH: Oriental medical hospital; OR: Odds ratio; RBC: Red blood cell; WBC: White blood cell count

\section{Acknowledgements}

None.

\section{Funding}

None.

\section{Availability of data and materials}

The datasets used and/or analysed during the current study are available from the corresponding author on reasonable request.

\section{Authors' contributions}

SYM, JSS, KHP and MSL were involved in the study design. SYM and KHP were involved in all data acquisition. SYM and JSS were involved in data analysis and interpretation, and drafted the manuscript. All authors read and approved the final manuscript.

\section{Ethics approval and consent to participate}

The study protocol was approved by institutional review board of Kyung Hee University Hospital at Gangdong (IRB No. 2016-03-008). Consent from the patients was waived by IRB, as the study was retrospective.

\section{Consent for publication}

Not applicable.

\section{Competing interests}

The authors declare that they have no competing interests.

\section{Publisher's Note}

Springer Nature remains neutral with regard to jurisdictional claims in published maps and institutional affiliations.

\section{Author details}

'Department of Internal Medicine, Kyung Hee University School of Medicine, Seoul, South Korea. ${ }^{2}$ Division of Infectious Diseases, Department of Internal Medicine, Kyung Hee University Hospital at Gangdong, 892, Dongnam-ro, Gangdong-gu, Seoul, South Korea.

Received: 4 May 2016 Accepted: 29 January 2018

Published online: 07 February 2018

\section{References}

1. Arbo MJ, Fine MJ, Hanusa BH, Sefcik T, Kapoor WN. Fever of nosocomial origin: etiology, risk factors, and outcomes. Am J Med. 1993;95(5):505-12.

2. Trivalle C, Chassagne P, Bouaniche M, Landrin I, Marie I, Kadri N, Menard JF, Lemeland JF, Doucet J, Bercoff E. Nosocomial febrile illness in the elderly: frequency, causes, and risk factors. Arch Intern Med. 1998;158(14):1560-5.

3. Chang FY, Singh N, Gayowski T, Wagener MM, Marino IR. Fever in liver transplant recipients: changing spectrum of etiologic agents. Clin Infect Dis. 1998;26(1):59-65.

4. Chanock SJ, Pizzo PA. Fever in the neutropenic host. Infect Dis Clin N Am. 1996:10(4):777-96

5. Donowitz GR. Fever in the compromised host. Infect Dis Clin N Am. 1996; 10(1):129-48.

6. Norman DC. Fever in the elderly. Clin Infect Dis. 2000;31(1):148-51.
7. Toussaint E, Bahel-Ball E, Vekemans M, Georgala A, Al-Hakak L, Paesmans M, Aoun M. Causes of fever in cancer patients (prospective study over 477 episodes). Support Care Cancer. 2006;14(7):763-9.

8. Kaul DR, Flanders SA, Beck JM, Saint S. Brief report: incidence, etiology, risk factors, and outcome of hospital-acquired fever: a systematic, evidencebased review. J Gen Intern Med. 2006;21(11):1184-7.

9. Kumar H, Song SY, More SV, Kang SM, Kim BW, Kim IS, Choi DK. Traditional Korean east Asian medicines and herbal formulations for cognitive impairment. Molecules (Basel, Switzerland). 2013;18(12):14670-93.

10. Garner JS, Jarvis WR, Emori TG, Horan TC, Hughes JM. CDC definitions for nosocomial infections, 1988. Am J Infect Control. 1988;16(3):128-40.

11. He XR, Wang Q, Li PP. Acupuncture and moxibustion for cancer-related fatigue: a systematic review and meta-analysis. Asian Pac J Cancer Prev. 2013;14(5):3067-74.

12. McCabe WR, Jackson G. Gram-negative bacteremia: I. Etiology and ecology. Arch Intern Med. 1962;110(6):847-55.

13. Cheung F. TCM: made in China. Nature. 2011;480(7378):S82-3.

14. Park HL, Lee HS, Shin BC, Liu JP, Shang Q, Yamashita H, Lim B. Traditional medicine in china, Korea, and Japan: a brief introduction and comparison. Evidence-based complementary and alternative medicine : eCAM. 2012; 2012:429103.

15. Rostami M, Mirmohammadsadeghi M, Zohrenia H. Evaluating the frequency of postoperative fever in patients with coronary artery bypass surgey. ARYA atherosclerosis. 2011;7(3):119-23.

16. Lee MS, Choi TY, Kang JW, Lee BJ, Ernst E. Moxibustion for treating pain: a systematic review. Am J Chin Med. 2010;38(5):829-38.

17. Cunha BA. Clinical approach to fever in the neurosurgical intensive care unit: focus on drug fever. Surg Neurol Int. 2013;4(Suppl 5):S318-22.

18. Cunha BA, Shea KW. Fever in the intensive care unit. Infect Dis Clin N Am. 1996;10(1):185-209.

19. Patel RA, Gallagher JC. Drug fever. Pharmacotherapy. 2010;30(1):57-69.
Submit your next manuscript to BioMed Central and we will help you at every step:

- We accept pre-submission inquiries

- Our selector tool helps you to find the most relevant journal

- We provide round the clock customer support

- Convenient online submission

- Thorough peer review

- Inclusion in PubMed and all major indexing services

- Maximum visibility for your research

Submit your manuscript at www.biomedcentral.com/submit 